\title{
基于 $\mathrm{Al}_{2} \mathrm{O}_{3}$ 介质的 $\mathrm{Ga}_{2} \mathrm{O}_{3} \mathrm{MOSFET}$ 器件制备研究
}

\author{
吕元杰，宋旭波，何泽召，谭 金銍，幸叶，王元刚，顾国栋，冯志红 \\ (河北半导体研究所, 专用集成电路国家级重点实验室, 石家庄 050051)
}

\begin{abstract}
摘 要: 采用金属有机化学气相沉积(MOCVD)方法在 $\mathrm{Fe}$ 掺杂半绝缘 $(010) \mathrm{Ga}_{2} \mathrm{O}_{3}$ 同质祄底上外延得到 $\mathrm{n}$ 型 $\beta-\mathrm{Ga}_{2} \mathrm{O}_{3}$ 薄膜材料, 材料结构包括 $600 \mathrm{~nm}$ 未掺杂的 $\mathrm{Ga}_{2} \mathrm{O}_{3}$ 缓冲层和 $200 \mathrm{~nm} \mathrm{Si}$ 掺杂沟道层。对掺杂浓度为 $3.0 \times 10^{17}$ 和 $1.0 \times$ $10^{18} \mathrm{~cm}^{-3}$ 的样品进行了高温合金欧姆接触实验, 在掺杂浓度为 $3.0 \times 10^{17} \mathrm{~cm}^{-3}$ 的样品上难以实现良好的欧姆接触, 掺 杂浓度为 $1.0 \times 10^{18} \mathrm{~cm}^{-3}$ 的样品实现了欧姆接触最低值 $(9.8 \Omega \cdot \mathrm{mm})$ 。基于掺杂浓度为 $1.0 \times 10^{18} \mathrm{~cm}^{-3}$ 的 $\mathrm{n}$ 型 $\beta-\mathrm{Ga}_{2} \mathrm{O}_{3}$ 薄 膜材料, 采用原子层沉积的 $\mathrm{Al}_{2} \mathrm{O}_{3}$ 作为棚下绝缘介质层, 研制出 $\mathrm{Ga}_{2} \mathrm{O}_{3}$ 金属氧化物半导体场效应晶体管(MOSFET)。 栅压为 $2 \mathrm{~V}$ 时, 器件漏源饱和电流达到 $108 \mathrm{~mA} / \mathrm{mm}$, 器件峰值跨导达到 $17 \mathrm{mS} / \mathrm{mm}$ 。由于栅漏电特性较差, 器件的 三端击穿电压仅为 $23 \mathrm{~V} @ V_{\mathrm{gs}}=-12 \mathrm{~V}$ 。采用高介电常数的 $\mathrm{HfO}_{2}$ 或者 $\mathrm{Al}_{2} \mathrm{O}_{3} / \mathrm{HfO}_{2}$ 复合结构作为栅下介质能够改善 棚漏电特性, 提升器件的击穿性能。
\end{abstract}

关 键 词: 氧化镓; 金属氧化物半导体场效应晶体管; 漏源饱和电流; 樄漏电 中图分类号: TQ174 文献标识码: A

\section{$\mathrm{Ga}_{2} \mathrm{O}_{3}$ MOSFET Device with $\mathrm{Al}_{2} \mathrm{O}_{3}$ Gate Dielectric}

\author{
LV Yuan-Jie, SONG Xu-Bo, HE Ze-Zhao, TAN Xin, ZHOU Xing-Ye, \\ WANG Yuan-Gang, GU Guo-Dong, FENG Zhi-Hong \\ (National Key Laboratory of ASIC, Hebei Semiconductor Research Institute, Shijiazhuang 050051, China)
}

\begin{abstract}
Ga}_{2} \mathrm{O}_{3}$ was homoepitaxially grown by metal organic chemical vapor deposition (MOCVD) on a Fe-doped semi-insulating (010) $\mathrm{Ga}_{2} \mathrm{O}_{3}$ substrate. The structure consisted of a $600 \mathrm{~nm}$ undoped (UID) $\mathrm{Ga}_{2} \mathrm{O}_{3}$ buffer layer and $200 \mathrm{~nm}$ Si-doped channel layer. High-temperature Ohmic alloy experiments were taken on two kinds of n-typed $\beta-\mathrm{Ga}_{2} \mathrm{O}_{3}$ with $\mathrm{Si}$ donor concentrations of $3.0 \times 10^{17}$ and $1.0 \times 10^{18} \mathrm{~cm}^{-3}$. It's hard to realize good Ohmic contact on the $\beta-\mathrm{Ga}_{2} \mathrm{O}_{3}$ epitaxial layer with donor concentrations of $3.0 \times 10^{17} \mathrm{~cm}^{-3}$. The lowest Ohmic value of $9.8 \Omega \cdot \mathrm{mm}$ was obtained on the substrate with donor concentrations of $1.0 \times 10^{18} \mathrm{~cm}^{-3}$. Metal-oxide-semiconductor field-effect transistor (MOSFET) was fabricated based on homoepitaxial $\beta$ - $\mathrm{Ga}_{2} \mathrm{O}_{3}$ film with donor concentrations of $1.0 \times 10^{18} \mathrm{~cm}^{-3}$, in which $\mathrm{Al}_{2} \mathrm{O}_{3}$ grown by atomic layer deposition (ALD) was used as gate dielectric. The drain saturation current of the fabricated device reached $108 \mathrm{~mA} / \mathrm{mm}$ at $V_{\mathrm{gs}}$ of $2 \mathrm{~V}$, and a high peak transconductance of $17 \mathrm{mS} / \mathrm{mm}$ was obtained. Due to poor gate leakage, the three-terminal off-state breakdown voltage was just $23 \mathrm{~V}$ at $V_{\mathrm{gs}}=-12 \mathrm{~V}$. The breakdown characteristics can be improved by introducing $\mathrm{HfO}_{2}$ with high dielectric constant or $\mathrm{Al}_{2} \mathrm{O}_{3} / \mathrm{HfO}_{2}$ composite structure.
\end{abstract}

Key words: $\mathrm{Ga}_{2} \mathrm{O}_{3}$; MOSFET; drain saturation current; gate leakage

收稿日期: 2017-11-09; 收到修改稿日期: 2018-01-04

基金项目: 国家自然科学基金(61674130, 61604137) National Natural Science Foundation of China (61674130, 61604137)

作者简介: 吕元杰(1985-), 男, 博士，副研究员. E-mail: yuanjielv@163.com

通讯作者: 冯志红, 研究员. E-mail: ga917vv@163.com 
氧化镓 $\left(\mathrm{Ga}_{2} \mathrm{O}_{3}\right)$ 材料是一种超宽禁带半导体, 比 氮化镓 $(\mathrm{GaN})$ 和碳化硅 $(\mathrm{SiC})$ 具有更为优越的物理特 性，成为近年来新型功率半导体材料与器件领域的 研究热点 ${ }^{[1-3]}$ 。氧化镓是一种III-VI族直接带隙宽禁 带半导体材料, 室温下禁带宽度可达到 $4.8 \mathrm{eV}$, 有 5 种不同的多形体结构, 其中 $\beta$ 相(单斜)结构最为稳 定, 其余四种为亚稳定结构。一般 $\mathrm{Ga}_{2} \mathrm{O}_{3}$ 薄膜由于 存在氧空位而呈现 $\mathrm{n}$ 型导电, 可以通过掺杂硅 $(\mathrm{Si})$ 和锡 $(\mathrm{Sn})$ 等杂质实现精确的 $\mathrm{n}$ 型掺杂，但 $\mathrm{p}$ 型 $\mathrm{Ga}_{2} \mathrm{O}_{3}$ 的制备仍然是一个世界性难题。 $\mathrm{Ga}_{2} \mathrm{O}_{3}$ 材料的击穿 电场强度可达 $8 \mathrm{MV} / \mathrm{cm}$, 是 $\mathrm{SiC}$ 和 $\mathrm{GaN}$ 材料理论击 穿场强的 3 倍左右, 非常适用于高击穿电压器件领 域。巴利加(Baliga)优值是用来全面评价半导体材料 在功率器件中的应用价值指数, 除与击穿电场强度 有关, 还会受到电子迁移率和介电常数的影响。 $\beta-\mathrm{Ga}_{2} \mathrm{O}_{3}$ 的巴利加优值是 $\mathrm{GaN}$ 的 4 倍、 $\mathrm{SiC}$ 的 10 倍 以上。因此, 在达到相同耐压的条件下, 采用 $\beta-\mathrm{Ga}_{2} \mathrm{O}_{3}$ 的单极器件在理论上的导通电阻仅为 $\mathrm{SiC}$ 的 $1 / 10 、 \mathrm{GaN}$ 的 $1 / 3$, 这有利于减少电源电路的导通 电力损耗, 最大程度上提高器件的功率密度。 $\beta-\mathrm{Ga}_{2} \mathrm{O}_{3}$ 在制备大功率密度的功率电子器件方面具 有得天独厚的优势。此外, $\mathrm{Ga}_{2} \mathrm{O}_{3}$ 单晶同质祄底为高 质量低缺陷 $\beta-\mathrm{Ga}_{2} \mathrm{O}_{3}$ 外延奠定了基础, 而且 $\mathrm{Ga}_{2} \mathrm{O}_{3}$ 单晶祄底可采用制备蓝宝石 $\left(\mathrm{Al}_{2} \mathrm{O}_{3}\right)$ 衬底同样的工 艺, 适合低成本的大批量生产, $\mathrm{Ga}_{2} \mathrm{O}_{3}$ 功率器件在制 备成本方面有明显优势 ${ }^{[4-6]}$ 。

目前国外已有很多关于 $\beta-\mathrm{Ga}_{2} \mathrm{O}_{3}$ 器件的报道, 如金属氧化物半导体场效应晶体管(MOSFETs), 金 属半导体场效应晶体管(MESFETs) 以及肖特基二极 管等 ${ }^{[7-8]}$, 并取得了不错的研究成果。Wong 等 ${ }^{[9]}$ 利用 分子束外延设备(MBE) 在 $\mathrm{Fe}$ 掺杂(010)取向的 $\mathrm{Ga}_{2} \mathrm{O}_{3}$ 单晶同质祄底上外延得到 $\mathrm{Si}$ 掺杂的 $\mathrm{n}$ 型 $\beta-\mathrm{Ga}_{2} \mathrm{O}_{3}$ 外 延薄膜, 并采用原子层沉积的 $\mathrm{Al}_{2} \mathrm{O}_{3}$ 作为栅下介质, 同时结合栅场板结构研制出 $\beta-\mathrm{Ga}_{2} \mathrm{O}_{3}$ MOSFET, 器 件漏源电流开关比达到 $10^{9}$, 击穿电压超过 $750 \mathrm{~V}$, 该值为目前 $\mathrm{Ga}_{2} \mathrm{O}_{3}$ MOSFET 器件报道最高值。Green 等 ${ }^{[1]}$ 采用金属有机气相外延(MOPVE) 方法在(010) 取向的 $\mathrm{Ga}_{2} \mathrm{O}_{3}$ 单晶同质祄底上外延得到 $\mathrm{Sn}$ 掺杂的 $\mathrm{n}$ 型 $\beta-\mathrm{Ga}_{2} \mathrm{O}_{3}$ 外延薄膜，同样采用原子层沉积的 $\mathrm{Al}_{2} \mathrm{O}_{3}$ 作为柱下介质, 研制出栅长为 $2 \mu \mathrm{m}$ 、栅源间距为 $0.8 \mu \mathrm{m}$ 、栅漏间距为 $0.6 \mu \mathrm{m}$ 的 $\beta-\mathrm{Ga}_{2} \mathrm{O}_{3}$ MOSFET, 器 件的击穿场强超过 $3.8 \mathrm{MV} / \mathrm{cm}$, 该值已经超过了 $\mathrm{GaN}(3 \mathrm{MV} / \mathrm{cm})$ 和 $\mathrm{SiC}(3.18 \mathrm{MV} / \mathrm{cm})$ 的理论击穿场 强。Zhou 等 ${ }^{[10]}$ 采用撕拉法从(100)取向的 $\mathrm{n}$ 型 $\mathrm{Ga}_{2} \mathrm{O}_{3}$ 祄底上转移获得纳米带状的 $\beta-\mathrm{Ga}_{2} \mathrm{O}_{3}$ 材料, 研制出背 栅结构的 $\beta-\mathrm{Ga}_{2} \mathrm{O}_{3}$ MOSFET, 饱和电流达到 $1.5 \mathrm{~A} / \mathrm{mm}$,
为目前饱和电流报道最高值, 而且该器件可以通过 改变撕拉薄膜的厚度实现阈值电压的调控, 阈值电 压能够达到 $100 \mathrm{~V}^{[11]}$, 但该方法获得的 $\mathrm{Ga}_{2} \mathrm{O}_{3}$ 材料 由于薄膜厚度等方面的限制，难以实际应用。

国外在 $\beta-\mathrm{Ga}_{2} \mathrm{O}_{3}$ 材料外延, 尤其是 MOSFET 器 件方面已经取得了可喜的研究成果, 但是国内关于 $\mathrm{Ga}_{2} \mathrm{O}_{3}$ 的报道仅限于单晶衬底、材料外延、探测器 以及少量的肖特基二极管等方面 ${ }^{[12-14]}$, 在 $\beta-\mathrm{Ga}_{2} \mathrm{O}_{3}$ MOSFET 器件制备方面还未有报道。本工作基于 $\mathrm{n}$ 型 $\beta-\mathrm{Ga}_{2} \mathrm{O}_{3}$ 外延薄膜材料, 成功研制了 $\beta-\mathrm{Ga}_{2} \mathrm{O}_{3}$ MOSFET 器件。

\section{1 材料外延与器件工艺}

\section{$1.1 \mathrm{n}$ 型 $\boldsymbol{\beta}-\mathrm{Ga}_{2} \mathrm{O}_{3}$ 薄膜外延}

图 1 为 $\mathrm{Ga}_{2} \mathrm{O}_{3}$ MOSFET 器件横截面结构示意 图。采用金属有机化学气相沉积(MOCVD) 设备在 $\mathrm{Fe}$ 掺杂半绝缘 $(010) \mathrm{Ga}_{2} \mathrm{O}_{3}$ 祄底上外延得到 $\beta-\mathrm{Ga}_{2} \mathrm{O}_{3}$ 外延薄膜。外延材料从衬底往上依次为 $600 \mathrm{~nm}$ 非 故意掺杂的 $\mathrm{Ga}_{2} \mathrm{O}_{3}$ 缓冲层和 $200 \mathrm{~nm} \mathrm{Si}$ 掺杂的沟道 层。这里制备了两种不同 $\mathrm{Si}$ 掺杂浓度的样品, 掺杂 浓度分别为 $3.0 \times 10^{17}$ 和 $1.0 \times 10^{18} \mathrm{~cm}^{-3}$ 。室温霍尔测 试表明，这两种材料的载流子迁移率分别为 42 和 $20 \mathrm{~cm}^{2} /(\mathrm{V} \cdot \mathrm{s})$ 。

\section{2 欧姆接触实验}

在两种掺杂浓度的样品上分别开展了欧姆接触 实验。欧姆接触电极采用 $\mathrm{Ti} / \mathrm{Au}(20 / 180 \mathrm{~nm})$ 两层金 属，随后利用快速退火设备在氮气氛围中退火 $1 \mathrm{~min}$, 退火温度分别为 $400^{\circ} \mathrm{C} 、 450^{\circ} \mathrm{C} 、 500^{\circ} \mathrm{C} 、 550^{\circ} \mathrm{C}$ 和 $600^{\circ} \mathrm{C}$ 。利用线性传输模型测量得到不同退火条件下

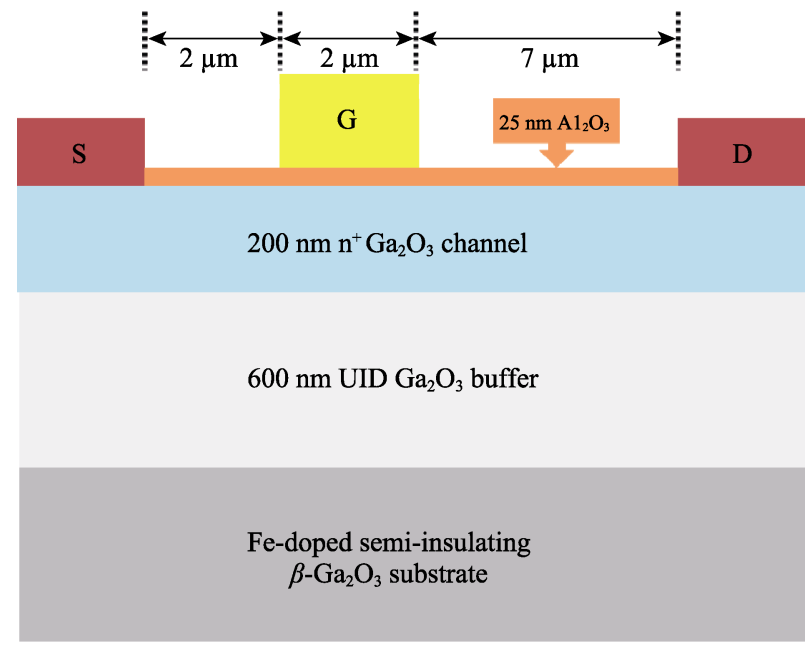

图 $1 \mathrm{Ga}_{2} \mathrm{O}_{3}$ MOSFET 器件横截面结构示意图

Fig. 1 Schematic cross section of the fabricated $\mathrm{Ga}_{2} \mathrm{O}_{3}$ MOSFET 
器件的欧姆接触值 $\left(R_{\mathrm{C}}\right)$, 测试结果列于图 2 。由于 $\mathrm{Ga}_{2} \mathrm{O}_{3}$ 材料超宽禁带的特性, $\mathrm{n}$ 型掺杂浓度较低时, 难以实现良好的欧姆接触。从图 2 可以看到, $\mathrm{n}$ 型掺 杂浓度为 $3.0 \times 10^{17} \mathrm{~cm}^{-3}$ 时, 在 $400 \sim 600^{\circ} \mathrm{C}$ 的退火范 围内, 欧姆接触电阻均大于 $35 \Omega \cdot \mathrm{mm}$, 欧姆接触很 差; 当掺杂浓度提高到 $1.0 \times 10^{18} \mathrm{~cm}^{-3}$ 后, 可以实现 相对较好的欧姆接触, $450^{\circ} \mathrm{C}$ 以下退火可以实现最低 欧姆接触电阻为 $9.8 \Omega \cdot \mathrm{mm}$ 。采用离子注入工艺, 在 欧姆接触区域形成重掺杂, 可以进一步降低欧姆接 触特性, 降低器件导通电阻。

\section{3 器件制备工艺}

基于欧姆接触实验结果, 本工作在 $\mathrm{Si}$ 掺杂浓度 为 $1.0 \times 10^{18} \mathrm{~cm}^{-3}$ 的 $\beta-\mathrm{Ga}_{2} \mathrm{O}_{3}$ 外延薄膜上制备 MOSFET 器件。器件制备工艺从台面刻蚀开始, 采用电感耦 合等离子体(ICP)刻蚀设备, 并利用 $\mathrm{BCl}_{3}$ 和 $\mathrm{Ar}$ 进行 干法刻蚀, 氧化镓薄膜刻蚀速率约为 $90 \mathrm{~nm} / \mathrm{min}$, 台 面刻蚀高度为 $300 \mathrm{~nm}$ 。源漏欧姆接触采用 $\mathrm{Ti} / \mathrm{Au}$ $(20 / 180 \mathrm{~nm})$ 两层金属, 随后在氮气氛围中 $450^{\circ} \mathrm{C}$ 下 退火 $1 \mathrm{~min}$ 。采用原子层沉积(ALD)设备在器件表面 生长厚度为 $25 \mathrm{~nm}$ 的 $\mathrm{Al}_{2} \mathrm{O}_{3}$ 介质, 外延生长温度为 $250^{\circ} \mathrm{C}$, 采用水 $\left(\mathrm{H}_{2} \mathrm{O}\right)$ 作为氧源。最后利用蒸发剥离 工艺制备栅长为 $2 \mu \mathrm{m}$ 的栅电极, 其采用 $\mathrm{Ni} / \mathrm{Au}$ 两层 金属。图 3 为 $\mathrm{Ga}_{2} \mathrm{O}_{3} \mathrm{MOSFE}$ 器件显微镜照片, 栅源 间距 $\left(L_{\mathrm{gg}}\right)$ 为 $2 \mu \mathrm{m}$, 栅漏间距 $\left(L_{\mathrm{gd}}\right)$ 为 $7 \mu \mathrm{m}$ 。

\section{2 器件特性分析}

图 4 为 $\mathrm{Ga}_{2} \mathrm{O}_{3}$ MOSFET 的直流输出特性曲线, 该曲线是利用半导体参数分析仪测量得到的。在输 出特性曲线测试过程中, 漏端偏压 $\left(V_{\mathrm{ds}}\right)$ 从 $0 \mathrm{~V}$ 施加 到 $20 \mathrm{~V}$, 步长为 $0.1 \mathrm{~V}$; 栅端偏压 $\left(V_{\mathrm{gs}}\right)$ 从 $2 \mathrm{~V}$ 施加至 $-12 \mathrm{~V}$, 步长为 $-2 \mathrm{~V}$ 。从图 4 可以看到, 器件展示出良 好的夹断特性。在椓偏压为 $2 \mathrm{~V}$ 下, 漏源饱和电流

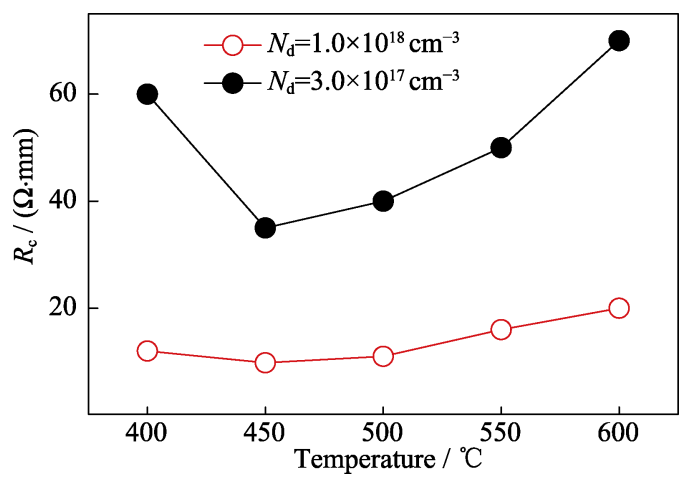

图 2 不同 $\mathrm{Si}$ 掺杂浓度下欧姆接触电阻随退火温度的变化

Fig. 2 Change of Ohmic contact resistances with annealing temperature at different $\mathrm{Si}$ donor concentrations

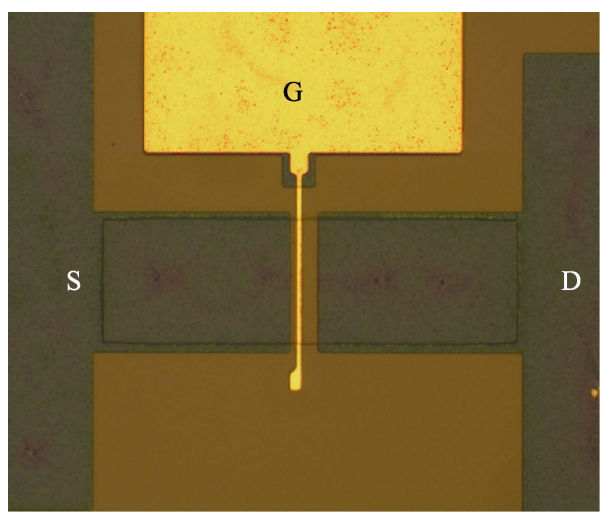

图 $3 \mathrm{Ga}_{2} \mathrm{O}_{3}$ MOSFET 器件显微镜照片

Fig. 3 Microscope image of the fabricated $\mathrm{Ga}_{2} \mathrm{O}_{3}$ MOSFET

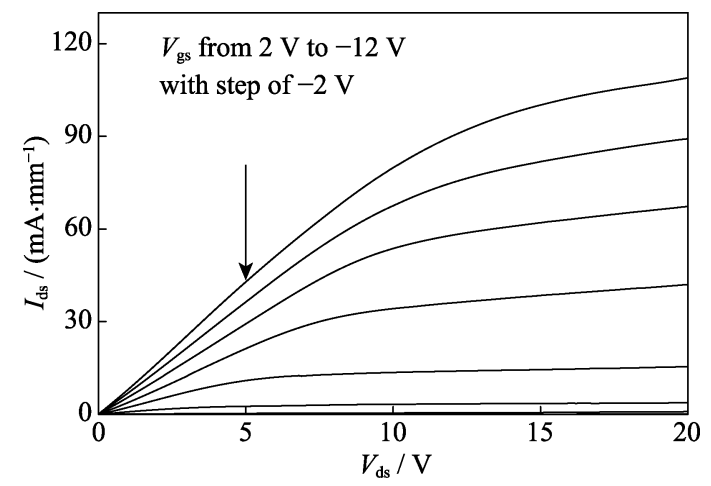

图 $4 \mathrm{Ga}_{2} \mathrm{O}_{3}$ MOSFET 器件输出特性曲线

Fig. 4 Output characteristics of the $\mathrm{Ga}_{2} \mathrm{O}_{3}$ MOSFET

达到 $108 \mathrm{~mA} / \mathrm{mm}$ 。从输出曲线外推得到开态导通电 阻 $\left(R_{\mathrm{ON}}\right)$ 为 $128.5 \Omega \cdot \mathrm{mm}$ 。表 1 汇总了已报道的基于 外延 $\mathrm{n}$ 型 $\mathrm{Ga}_{2} \mathrm{O}_{3}$ 薄膜的 MOSFET 相关电学参数, 本 研究外延的 $\mathrm{n}$ 型 $\mathrm{Ga}_{2} \mathrm{O}_{3}$ 薄膜材料在掺杂浓度为 $1.0 \times 10^{18} \mathrm{~cm}^{-3}$ 时, 载流子迁移率仅为 $20 \mathrm{~cm}^{2} /(\mathrm{V} \cdot \mathrm{s})$, 远低于国外已报道的结果, 还需继续优化外延工艺, 降低电离杂质散射, 提升载流子迁移率。此外, 相比 于已报道的 $\mathrm{Ga}_{2} \mathrm{O}_{3}$ MOSFET 器件, 本工作研制的器

表 $1 \mathrm{Ga}_{2} \mathrm{O}_{3}$ MOSFET 电学参数汇总

Table 1 Summary of electrical parameters for $\mathrm{Ga}_{2} \mathrm{O}_{3}$ MOSFET

\begin{tabular}{cccccc}
\hline & $\begin{array}{c}\text { Doping } \\
\text { concentra- } \\
\text { tion/cm }\end{array}$ & $\begin{array}{c}\text { Carrier } \\
\mathrm{mobility} / \\
\left(\mathrm{cm}^{2} \cdot \mathrm{V}^{-1} \cdot \mathrm{s}^{-1}\right)\end{array}$ & $\begin{array}{c}\text { Drain source } \\
\text { saturation } \\
\text { current/ } \\
\left(\mathrm{mA} \cdot \mathrm{mm}^{-1}\right)\end{array}$ & $\begin{array}{c}\text { Break- } \\
\text { down } \\
\text { voltage/V }\end{array}$ & Ref. \\
\hline 1 & $3.0 \times 10^{17}$ & $\mathrm{NR}$ & 26 & 370 & {$[4]$} \\
2 & $4.8 \times 10^{17}$ & 19.7 & 60 & 200 & {$[1]$} \\
3 & $3.0 \times 10^{17}$ & $70-95$ & 78 & 755 & {$[9]$} \\
4 & $2.3 \times 10^{17}$ & 24 & 0.24 & 612 & {$[11]$} \\
5 & $4.0 \times 10^{17}$ & 111 & 80 & 479 & {$[5]$} \\
6 & $1.3 \times 10^{18}$ & 96 & 150 & $\mathrm{NR}$ & {$[15]$} \\
\hline & $3.0 \times 10^{17}$ & 42 & & & This \\
& $1.0 \times 10^{18}$ & 20 & 108 & 23 & work \\
\hline
\end{tabular}


件展示出较高的漏源饱和电流密度, 这主要是 $\mathrm{n}$ 型 $\mathrm{Ga}_{2} \mathrm{O}_{3}$ 薄膜材料的掺杂浓度较高导致的。掺杂浓度 越高对器件的漏源饱和电流以及导通电阻越有利, 但会导致击穿特性变差。

图 5 给出了 $\mathrm{Ga}_{2} \mathrm{O}_{3}$ MOSFET 的转移特性曲线。 该测试曲线是利用半导体参数分析仪测量得到的, 测试过程中漏偏压固定为 $18 \mathrm{~V}$, 栅偏压从-14 V 增 加到 $2 \mathrm{~V}$, 步长为 $0.1 \mathrm{~V}$ 。器件的阈值电压 $\left(V_{\mathrm{th}}\right)$ 约为 $-10 \mathrm{~V}$, 峰值跨导达到 $17 \mathrm{mS} / \mathrm{mm}$ 。

图 6 为夹断状态下器件击穿特性, 测试过程中 栅压固定为 $-12 \mathrm{~V}$, 同时监测漏源电流 $\left(I_{\mathrm{ds}}\right)$ 和栅漏电 流 $\left(I_{\mathrm{g}}\right)$ 。从图 6 可以看到, 器件的三端击穿电压为 $23 \mathrm{~V}$, 此外还可以看到器件的栅漏电较大, 这是导 致器件击穿特性差的主要原因。在器件制备过程中 同时制备了金属 $/ \mathrm{Al}_{2} \mathrm{O}_{3} /$ 金属的电容结构, 发现该结 构的绝缘特性良好, 两端电流为 $10^{-11} \mathrm{~A}$ 量级, 击穿 电压大于 $200 \mathrm{~V}$ 。因此, 本工作生长的 $\mathrm{Al}_{2} \mathrm{O}_{3}$ 介质具 有良好的绝缘特性。器件栅漏电较大可能是由于 $\mathrm{Al}_{2} \mathrm{O}_{3}$ 与 $\mathrm{Ga}_{2} \mathrm{O}_{3}$ 的界面态导致的。据文献报道, 采用 $\mathrm{H}_{2} \mathrm{O}$ 作为氧源制备的 $\mathrm{Al}_{2} \mathrm{O}_{3}$ 介质容易在异质界面处 产生高浓度的固定正电荷和类受主界面态，从而导 致栅漏电较大 ${ }^{[16]}$ 。表 1 汇总的 $\mathrm{Ga}_{2} \mathrm{O}_{3}$ MOSFET 器件

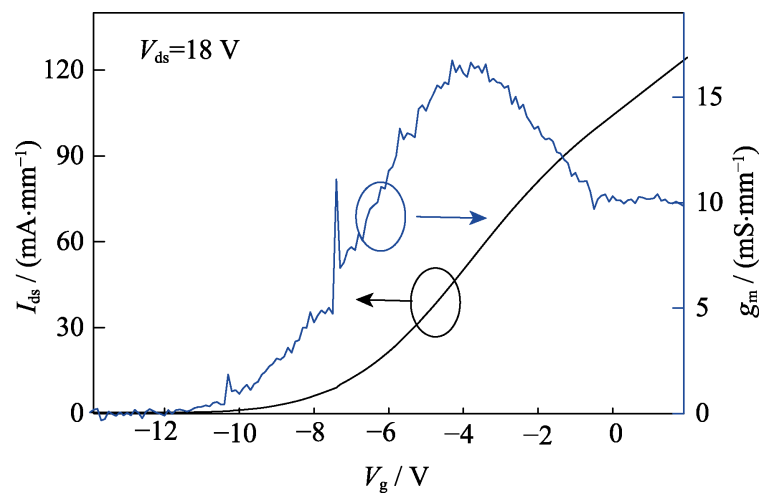

图 $5 \mathrm{Ga}_{2} \mathrm{O}_{3}$ MOSFE 器件的转移特性曲线

Fig. 5 Transfer characteristics of the $\mathrm{Ga}_{2} \mathrm{O}_{3}$ MOSFET

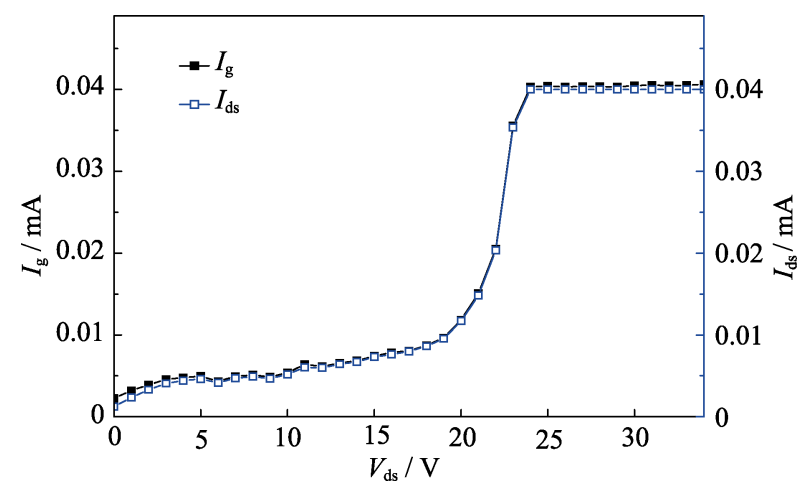

图 $6 \mathrm{Ga}_{2} \mathrm{O}_{3}$ MOSFE 器件的三端击穿特性曲线

Fig. 6 Three-terminal off-state breakdown characteristics for the $\mathrm{Ga}_{2} \mathrm{O}_{3}$ MOSFET
都是采用 $\mathrm{Al}_{2} \mathrm{O}_{3}$ 作为栅下介质, 且实现了很高的击 穿特性。因此，优化原子层沉积 $\mathrm{Al}_{2} \mathrm{O}_{3}$ 介质工艺，降 低界面态是下一步的工作重点。同时，将采用高介 电常数的 $\mathrm{HfO}_{2}$ 介质或者 $\mathrm{Al}_{2} \mathrm{O}_{3} / \mathrm{HfO}_{2}$ 复合结构作栅 下介质层降低柱漏电，以提升器件击穿特性。

\section{3 结论}

基于同质外延的 $\mathrm{n}$ 型 $\beta-\mathrm{Ga}_{2} \mathrm{O}_{3}$ 薄膜材料研制出了 $\mathrm{Ga}_{2} \mathrm{O}_{3}$ 金属氧化物半导体场效应晶体管(MOSFET)。 $\mathrm{n}$ 型 $\beta-\mathrm{Ga}_{2} \mathrm{O}_{3}$ 薄膜材料是在铁掺杂的半绝缘 $(010)$ $\mathrm{Ga}_{2} \mathrm{O}_{3}$ 祄底上同质外延实现的, 材料结构包括 $600 \mathrm{~nm}$ 未掺杂的 $\mathrm{Ga}_{2} \mathrm{O}_{3}$ 缓冲层和 $200 \mathrm{~nm}$ 硅掺杂的 $\mathrm{Ga}_{2} \mathrm{O}_{3}$ 沟道层, 其中沟道层 $\mathrm{n}$ 型掺杂浓度为 $1.0 \times 10^{18} \mathrm{~cm}^{-3}$ 。 $\mathrm{Ga}_{2} \mathrm{O}_{3}$ MOSFET 器件采用 $25 \mathrm{~nm} \mathrm{Al}_{2} \mathrm{O}_{3}$ 作为栅下绝 缘介质层, 研制的器件漏源饱和电流为 $108 \mathrm{~mA} / \mathrm{mm}$ @ $V_{\mathrm{gs}}=2 \mathrm{~V}$, 峰值跨导达到 $17 \mathrm{mS} / \mathrm{mm}$ 。由于栅漏电特 性较差, 导致器件的三段击穿电压仅为 $23 \mathrm{~V} @ V_{\mathrm{gs}}=$ $-12 \mathrm{~V}$ 。通过引入高 $\mathrm{K}$ 介质，如 $\mathrm{HfO}_{2}$ 或 $\mathrm{Al}_{2} \mathrm{O}_{3} / \mathrm{HfO}_{2}$ 复合结构作栅下介质层来改善漏电特性能够进一步 提升器件的击穿特性。

\section{参考文献:}

[1] GREEN A J, CHABAK K D, HELLER E R, et al. 3.8-MV/cm breakdown strength of MOVPE-grown Sn-doped $\beta-\mathrm{Ga}_{2} \mathrm{O}_{3}$ MOSFETs. IEEE Electron Deivce Lett., 2016, 37(7): 902-905.

[2] ZHOU H, ALGHMADI S, SI M, et al. $\mathrm{Al}_{2} \mathrm{O}_{3} / \mathrm{Ga}_{2} \mathrm{O}_{3}(-201)$ interface improvement through piranha pretreatment and postdeposition annealing. IEEE Electron Deivce Lett., 2016, 37(11): 1411-1414.

[3] AHN S, REN F, KIM J, et al. Effect of front and back gates on $\beta$ - $\mathrm{Ga}_{2} \mathrm{O}_{3}$ nano-belt field-effect transistors. Applied Physics Letters, 2016, 109(6): 062102-1-4.

[4] HIGASHIWAKI M, SASAKI K, KURAMATA T, et al. Depletionmode $\mathrm{Ga}_{2} \mathrm{O}_{3}$ metal-oxide-semiconductor field-effect transistors on $\beta-\mathrm{Ga}_{2} \mathrm{O}_{3}(010)$ substrates and temperature dependence of their device characteristics. Applied Physics Letters, 2013, 103(12): $123511-1-4$.

[5] MOSER N, MCCANDLESS J, CRESPO A, et al. Ge-doped $\beta-\mathrm{Ga}_{2} \mathrm{O}_{3}$ MOSFETs. IEEE Electron Deivce Lett., 2017, 38(6): 775-778.

[6] ZHOU H, SI M, ALGHAMADI S, et al. High-performance depletion/ enhancement-mode $\beta-\mathrm{Ga}_{2} \mathrm{O}_{3}$ on insulator (GOOI) field-effect transistors with record drain currents of $600 / 450 \mathrm{~mA} / \mathrm{mm}$. IEEE Electron Deivce Lett., 2017, 38(1): 103-106.

[7] HIGASHIWAKI M, SASAKI K, KURAMATA A, et al. Gallium oxide $\left(\mathrm{Ga}_{2} \mathrm{O}_{3}\right)$ metal-semiconductor field-effect transistors on singlecrystal $\beta-\mathrm{Ga}_{2} \mathrm{O}_{3}$ (010) substrates. Appl. Phys. Lett., 2012, 100(1): 013504-1-3. 
[8] SASAKI K, HIGASHIWAKI M, KURAMATA A, et al. $\mathrm{Ga}_{2} \mathrm{O}_{3}$ Schottky barrier diodes fabricated by using single-crystal $\beta-\mathrm{Ga}_{2} \mathrm{O}_{3}$ (010) substrates. IEEE Electron Deivce Lett., 2013, 34(4): 493-495.

[9] WONG M H, SASAKI K, KURAMATA A, et al. Field-plated $\mathrm{Ga}_{2} \mathrm{O}_{3}$ MOSFET with a breakdown voltage of over $750 \mathrm{~V}$. IEEE Electron Deivce Lett., 2016, 37(2): 212-215.

[10] ZHOU H, MAIZE K, QIU G, et al. $\beta-\mathrm{Ga}_{2} \mathrm{O}_{3}$ on insulator field-effect transistors with drain currents exceeding $1.5 \mathrm{~A} / \mathrm{mm}$ and their self-heating effect. Appl. Phys. Lett., 2017, 111(9): 092102$1-4$.

[11] CHABAK K D, MOSER N, GREEN A J, et al. Enhanced-mode $\mathrm{Ga}_{2} \mathrm{O}_{3}$ wrap-gate fin field-effect transistors on native (010) $\beta-\mathrm{Ga}_{2} \mathrm{O}_{3}$ substrate with high breakdown voltage. Appl. Phys. Lett., 2016, 109(21): 213501-1-5.

[12] GUO JIN-JIN, LIU AI-HUA, MAN BAO-HUA, et al. Heteroepti- taxy, ultraviolaet and luminescence characterizations of $\beta-\mathrm{Ga}_{2} \mathrm{O}_{3}$ grown using LMBE. Materials Review, 2012, 26(6): 52-58.

[13] HE BIN, XING JIE, DUAN YAN-TING, et al. Research progress of $\mathrm{Ga}_{2} \mathrm{O}_{3}$ UV photodetectors. Materials Review, 2013, 27(22): 157-163.

[14] ZHANG HONG-ZHE, WANG LIN-JUN, XIA CHANG-TAI, et al. Research progress of wide-gap semiconductor $\beta-\mathrm{Ga}_{2} \mathrm{O}_{3}$ single crystal. Journal of Synthetic Crystals, 2015, 44(11): 2952-2973.

[15] GREEN A J, CHABAK K D, BALDINI M, et al. $\beta-\mathrm{Ga}_{2} \mathrm{O}_{3}$ MOSFETs for radio frequency operation. IEEE Electron Deivce Lett., 2017, 38(6): 790-793.

[16] HUANG S, YANG S, ROBERTS J, et al. Threshold voltage instability in $\mathrm{Al}_{2} \mathrm{O}_{3} / \mathrm{GaN} / \mathrm{AlGaN} / \mathrm{GaN}$ metal-insulator-semiconductor high-electron mobility transistors. Jpn. J. Appl. Phys., 2011, 50(11): 110202-1-3. 